\title{
FATOU-TYPE THEOREMS FOR GENERAL APPROXIMATE IDENTITIES
}

\author{
MARCUS CARLSSON
}

\begin{abstract}
For functions $f \in L^{1}\left(\mathbf{R}^{n}\right)$ we consider extensions to $\mathbf{R}^{n} \times \mathbf{R}^{+}$given by convolving $f$ with an approximate identity. For a large class of approximate identities we obtain a Fatou-type theorem where the convergence regions are sometimes effectively larger than the non-tangential ones. We then study a more restricted class of approximate identities for which the convergence regions are shown to be optimal. Finally we will consider products of approximate identities. The results extend previous results by Sjögren [4], Rönning [2] and Brundin [1].
\end{abstract}

\section{Introduction}

For a function $f \in L^{1}(\mathrm{R})$, let $\hat{f}$ denote its harmonic extension to the upper half plane, i.e.

$$
\hat{f}(x, r)=\int_{\mathrm{R}} P(x-t, r) f(t) d t
$$

where

$$
P(x, r)=\frac{r}{\pi\left(x^{2}+r^{2}\right)}
$$

is the Poisson kernel on R. The classical Fatou theorem asserts that for a.e. $\theta \in \mathrm{R}$, the harmonic extension $\hat{f}(x, r)$ has the limit $f(\theta)$ when $(x, r) \rightarrow(\theta, 0)$ within the angle

$$
\mathscr{A}(\theta)=\{(x, r):|x-\theta|<a r\},
$$

where $a>0$ is any fixed constant.

The Poisson kernel is a special case of an approximate identity. In general, these are defined as follows.

Definition 1.1. An approximate identity on $\mathrm{R}^{n}$ is a function $P: \mathrm{R}^{n} \times$ $\mathrm{R}^{+} \rightarrow \mathrm{R}^{+}$such that

$$
\|P(\cdot, r)\|_{1}=1 \quad \text { for all } \quad r>0 .
$$

Received March 21, 2006; in revised form September 27, 2006. 
For any open set $\Omega$ containing $0, P(\cdot, r)$ converge to 0 in both

$$
L^{\infty}\left(\mathrm{R}^{n} \backslash \Omega\right) \quad \text { and } \quad L^{1}\left(\mathrm{R}^{n} \backslash \Omega\right), \quad \text { as } \quad r \rightarrow 0 .
$$

Using any approximate identity $P$, we may extend a function $f \in L^{1}\left(\mathrm{R}^{n}\right)$ to a function $\hat{f}$ on $\mathbf{R}^{n} \times \mathbf{R}^{+}$as in the formula (1.1). The question wether Fatou's theorem continues to hold for other approximate identities has been thoroughly investigated and plays an important role in harmonic analysis, especially in the theory of Hardy spaces. The special types of approximate identities which occur in this context arise by dilation of functions on $\mathrm{R}^{+}$(see [5], p. 23-24), that is, approximate identities of the type

$$
P_{h}(x, r)=\frac{1}{r^{n}} h\left(\frac{|x|}{r}\right),
$$

where $|\cdot|$ denotes the Euclidean distance in $\mathrm{R}^{n}$ and $h$ is some positive decreasing $L^{1}\left(\mathrm{R}^{+}\right)$-function such that $\left\|P_{h}(\cdot, r)\right\|_{1}=1$. By ([5], p. 57), it follows that Fatou's theorem continues to hold for any approximate identity $P$ which is bounded above by an approximate identity $P_{h}$ as in (1.4). More precisely, for such $P$, any $f \in L^{1}\left(\mathrm{R}^{n}\right)$ and a.e. $\theta \in \mathrm{R}^{n}$, the function

$$
\hat{f}(x, r)=(P(\cdot, r) * f)(x)=\int_{\mathrm{R}^{n}} P(x-t, r) f(t) d \lambda(t)
$$

has the limit $f(\theta)$ when $r \rightarrow 0$ and $|x-\theta|<a r$, where $a>0$ is any fixed constant and $\lambda$ denotes the Lebesgue measure on $\mathrm{R}^{n}$.

An interesting phenomenon related to these results has been observed by Sjögren [4]. Let D denote the unit disc and let $\mathrm{T}$ denote its boundary.

Let us consider approximate identities on the unit circle obtained by normalizing powers of the usual Poisson kernel, that is, approximate identities of the form

$$
P_{\alpha}\left(e^{i t}, r\right)=\frac{\left(P\left(e^{i t}, r\right)\right)^{\alpha}}{\left\|(P(\cdot, r))^{\alpha}\right\|_{1}},
$$

where $1 / 2 \leq \alpha \leq 1$ and $P\left(e^{i t}, r\right)=\frac{1-r^{2}}{\left|e^{i t}-r\right|^{2}}$. If $\alpha>1 / 2$, then using the previously mentioned result about approximate identities of the form (1.4) one can show that Fatou's theorem continues to hold in this case as well, i.e. given $f \in L^{1}(\mathrm{~T})$ the function $\hat{f}$ defined on $\mathrm{D}$ by

$$
\hat{f}\left(r e^{i t}\right)=\int_{0}^{2 \pi} P_{\alpha}\left(e^{i(s-t)}, r\right) f\left(e^{i s}\right) \frac{d s}{2 \pi}
$$

has the limit $f(\xi)$ when $r e^{i t} \rightarrow \xi$ non-tangentially for a.e. $\xi \in \mathrm{T}$. However, in the limit case when $\alpha=1 / 2$, the approximate identity $P_{1 / 2}$ cannot be 
treated with these methods. Sjögren [4] was the first to observe that for $P_{1 / 2}$ we actually get larger regions of convergence. Later Rönning [2] improved this for functions $f \in L^{p}(\mathbf{T})$ with $p>1$, and showed that these regions increase with $p$. Their results can be summarized in the following theorem.

TheOREm 1.2. Let $f \in L^{p}(\mathrm{~T})$ where $1 \leq p<\infty$ and set $\rho(r)=$ $(1-r)\left(\log \frac{1}{1-r}\right)^{p}$. Then for a.e. $\xi \in \mathrm{T}$ the function

$$
\hat{f}\left(r e^{i t}\right)=\int_{0}^{2 \pi} P_{1 / 2}\left(e^{i(t-s)}, r\right) f\left(e^{i s}\right) \frac{d s}{2 \pi}
$$

has the limit $f(\xi)$ when $r e^{i t} \rightarrow \xi$ inside the region

$$
\mathscr{A}(\xi)=\left\{r e^{i t}:|\arg \xi-t|<a \rho(r)\right\}
$$

for any fixed constant a. Moreover, these regions are optimal in the sense that if $\rho$ is any function for which the above conclusion holds, then $\rho(r)=$ $O\left((1-r)\left(\log \frac{1}{1-r}\right)^{p}\right)$ when $r \rightarrow 1^{-}$. (O stands for "big ordo".)

The arguments given in [4] and [2] are quite involved and recently Brundin [1] has obtained a simpler proof of these results. However, all proofs are rather long and are essentially based on the specific formula defining $P_{1 / 2}$.

It is the purpose of this paper to show that this type of result continues to hold for any approximate identity whose level sets are sufficiently regular. This general point of view not only yields Fatou-type theorems and maximal function estimates for a large class of approximate identities which covers all examples mentioned above, but also leads to considerably shorter proofs, which, in some sense are a refinement of the approach used in the theory of Hardy spaces (see [5]). The regularity assumption which we are going to use has a simple formulation in geometrical terms, namely that the Lebesgue measure of each level set of the approximate identity $P$ is bounded below by a constant multiple of the measure of the smallest ball centered at 0 that includes the level set. In other words, if we set

$$
L(r, s)=\left\{x \in \mathrm{R}^{n}: P(x, r)>s\right\}
$$

then our condition is

$$
\sup \left\{|x|^{n}: x \in L(r, s)\right\} \leq c \lambda(L(r, s)) .
$$

for some constant $c$ and all $r, s$.

Throughout the paper, $p$ will be a fixed number with $1 \leq p<\infty$ and $q$ will be its conjugate index defined by $\frac{1}{p}+\frac{1}{q}=1$. The main theorem reads as follows. 
Theorem 1.3. Let $f \in L_{l o c}^{p}\left(\mathrm{R}^{n}\right) \cap L^{1}\left(\mathrm{R}^{n}\right)$. Let $P$ be an approximate identity such that (1.5) holds and set

$$
\rho(r)=\frac{1}{\|P(\cdot, r)\|_{q}^{p / n}}
$$

Then for a.e. $\theta \in \mathbf{R}^{n}$ the function

$$
\hat{f}(x, r)=(P(\cdot, r) * f)(x)=\int_{\mathrm{R}^{n}} P(x-t, r) f(t) d \lambda(t)
$$

has the limit $f(\theta)$ when $r \rightarrow 0$ and $x \rightarrow \theta$ inside the region

$$
\mathscr{A}(\theta)=\{(x, r):|x-\theta|<a \rho(r)\},
$$

where $a>0$ is any fixed constant.

The proof of Theorem 1.3, as well as a number of extensions, will be given in section 2.

In section 3 we consider a special class of approximate identities that contain all previous examples and for which our results are sharp, in the sense that the regions of convergence provided by Theorem 1.3 are optimal.

These approximate identities include those of type (1.4). They are defined in terms of a single positive function $h$ on $\mathrm{R}^{+}$and a number $0<R \leq \infty$, and the corresponding approximate identity is denoted by $P_{h, R}$. We shall show that the regions of convergence are completely determined by the function

$$
\iota(r)=\int_{0}^{R / r} h(s) s^{n-1} d s .
$$

More specifically, we will show that for each $f \in L_{l o c}^{p}\left(\mathrm{R}^{n}\right) \cap L^{1}\left(\mathrm{R}^{n}\right)$ and a.e. $\theta \in \mathbf{R}^{n}$ the function

$$
\hat{f}(x, r)=\left(P_{h, R}(\cdot, r) * f\right)(x)=\int_{\mathrm{R}^{n}} P_{h, R}(x-t, r) f(t) d \lambda(t)
$$

has the limit $f(\theta)$ when $r \rightarrow 0$ and $x \rightarrow \theta$ inside the region

$$
\mathscr{A}(\theta)=\left\{(x, r):|x-\theta|<\operatorname{arl}^{p / n}(r)\right\}
$$

where $a$ is any fixed constant. We will also show that these regions are optimal in the sense that if $\gamma$ is any function such that the above result holds at a.e. $\theta$ with $a r \iota^{p / n}(r)$ replaced with $\gamma$, then $\gamma(r)=O\left(r l^{p / n}(r)\right)$ when $r \rightarrow 0$. 
In particular, Theorem 1.2 is a direct consequence of these results and in general we see that the phenomenon observed by Sjögren occurs if and only if $R<\infty$ and

$$
\int_{0}^{\infty} h(s) s^{n-1} d s=\infty .
$$

At the end of section 3 we construct an example related to condition (1.5). It is not hard to see that the conclusion of Theorem 1.3 cannot hold for all approximate identities, but an interesting question is wether (1.5) can be removed for the approximate identities $P_{h, R}$ discussed above. We will show that the answer is negative.

Finally, in section 4 we consider the boundary behavior of convolutions with products of approximate identities. More specifically, let $P_{1}, \ldots, P_{n}$ be approximate identities on $\mathrm{R}$ and set

$$
P\left(\left(x_{1}, \ldots, x_{n}\right),\left(r_{1}, \ldots, r_{n}\right)\right)=P_{1}\left(x_{1}, r_{1}\right) \ldots P_{n}\left(x_{n}, r_{n}\right) .
$$

For functions $f \in L^{p}\left(\mathrm{R}^{n}\right)$ we will investigate the boundary behavior of

$$
\hat{f}(x, r)=\int_{\mathrm{R}^{n}} P(x-t, r) f(t) d \lambda(t) .
$$

This was studied by Zygmund (see [6], Ch. XVII) in the case when all $P_{i}$ 's are equal to the Poisson kernel and more recently Brundin [1] studied the corresponding problem with $P_{i}=P_{1 / 2}$ and $f \in L^{\infty}\left(\mathrm{T}^{n}\right)$.

Note that $P$ is not an approximate identity and moreover the level sets of $P$ do not satisfy a condition like (1.5) even in the case when the approximate identities $P_{i}$ equal the Poisson kernel.

As an application of the main theorem of section 4 we will extend Theorem 1.2 in the following way: Let $P: \mathrm{T}^{n} \times[0,1)^{n} \rightarrow \mathrm{R}^{+}$be given by

$$
P(\tau, r)=P_{1 / 2}\left(\tau_{1}, r_{1}\right) \ldots P_{1 / 2}\left(\tau_{n}, r_{n}\right)
$$

and the corresponding extension to $\mathrm{D}^{n}$ of a function $f \in L^{p}\left(\mathrm{~T}^{n}\right)$ be given by

$$
\begin{aligned}
\hat{f}\left(\left(r_{1} e^{i t_{1}}, \ldots, r_{n} e^{i t_{n}}\right)\right) & \\
& =\int_{[0,2 \pi]^{n}} P\left(\left(e^{i\left(t_{1}-s_{1}\right)}, \ldots, e^{i\left(t_{n}-s_{n}\right)}\right), r\right) f\left(\left(e^{i s_{1}}, \ldots, e^{i s_{n}}\right)\right) \frac{d s_{1} \ldots d s_{n}}{(2 \pi)^{n}}
\end{aligned}
$$

We then have:

THEOREM 1.4. Let $1 \leq p<\infty, \epsilon>0$ and $f \in L^{p+\epsilon}\left(\mathrm{T}^{n}\right)$ be given. Let $a>0$ be arbitrary and set

$$
\rho(r)=a(1-r)\left(\log \frac{1}{1-r}\right)^{p} .
$$


Then, for a.e. $\xi \in \mathrm{T}^{n}, \hat{f}(z) \rightarrow f(\xi)$ when $z \rightarrow \xi$ inside

$$
\mathscr{A}(\xi)=\left\{\left(r_{1} e^{i t_{1}}, \ldots, r_{n} e^{i t_{n}}\right) \in \mathrm{D}^{n}:\left|\arg \xi_{k}-t_{k}\right|<\rho\left(r_{k}\right)\right\} .
$$

This result is new and should be compared with Theorem 2 of Brundin [1], where the same result is proved under the assumption that $f \in L^{\infty}\left(\mathrm{T}^{n}\right)$ and that $\rho$ satisfies $\rho(r)=O\left((1-r)^{\alpha}\right)$ for all $\alpha<1$.

\section{Main results}

Throughout this paper $p$ will be a fixed number with $1 \leq p<\infty$ and $q$ will be the conjugate index defined by the equation $\frac{1}{p}+\frac{1}{q}=1$. Also, for $L \subset \mathrm{R}^{n}$ let $|L|=\sup \{|x|: x \in L\}$. Let $P$ be an approximate identity on $\mathrm{R}^{n}$. As before we let $\rho: \mathbf{R}^{+} \rightarrow \mathbf{R}^{+}$be the function

$$
\rho(r)=\frac{1}{\|P(\cdot, r)\|_{q}^{p / n}},
$$

and we define $L(r, s)=\left\{x \in \mathrm{R}^{n}: P(x, r)>s\right\}$. To obtain more generality, we shall work with the following assumption which is weaker than (1.5). It essentially says that for fixed $r,(1.5)$ holds for all $s$ in a certain interval.

There exist positive constants $c_{1}, c_{2}, c_{3}, c_{4}$ and a function

$$
\begin{aligned}
& u: \mathrm{R}^{+} \rightarrow \mathrm{R}^{+} \text {such that }|L(r, u(r))| \leq c_{1} \rho(r) \text { and }|L(r, s)|^{n} \leq \\
& c_{2} \lambda(L(r, s)) \text { holds for all } s \text { with } u(r)>s>c_{3} \text { and } r<c_{4} .
\end{aligned}
$$

The main theorem is the following result.

Theorem 2.1. Let $f \in L_{l o c}^{p}\left(\mathrm{R}^{n}\right) \cap L^{1}\left(\mathrm{R}^{n}\right), 1 \leq p<\infty$, and let $P$ be an approximate identity such that (2.2) holds. Then for a.e. $\theta \in \mathbf{R}^{n}$ the function

$$
\hat{f}(x, r)=(P(\cdot, r) * f)(x)=\int_{\mathrm{R}^{n}} P(x-t, r) f(t) d \lambda(t)
$$

has the limit $f(\theta)$ when $r \rightarrow 0$ and $x \rightarrow \theta$ inside the region

$$
\mathscr{A}(\theta)=\{(x, r):|x-\theta|<a \rho(r)\},
$$

where $a$ is any fixed constant.

Observe that we necessarily have $\lim _{r \rightarrow 0} \rho(r)=0$. To see this, note that by (1.3) and Hölder's inequality we have that for each open set $\Omega$ containing zero the inequality

$$
1 / 2 \leq \int_{\Omega} P(\cdot, r) d \lambda \leq(\lambda(\Omega))^{1 / p}\|P(\cdot, r)\|_{q}
$$


holds for sufficiently small $r$, which implies that for such $r$ we have $\rho(r) \leq$ $\left(2^{p} \lambda(\Omega)\right)^{1 / n}$.

As in the classical case of the Fatou theorem one can either give a direct proof of the result, or deduce it from an estimate of the corresponding maximal functions. We will give the latter proof.

With notation as in Theorem 2.1 we define the maximal function

$$
f^{*}(\theta)=\sup _{(x, r) \in \mathscr{A}(\theta)}|\hat{f}(x, r)|
$$

and let $f_{H L}$ denote the standard Hardy-Littlewood maximal function, i.e.

$$
f_{H L}(\theta)=\sup _{r>0} \frac{\int_{B(\theta, r)}|f| d \lambda}{\lambda(B(\theta, r))},
$$

where $B(\theta, r)$ is the ball centered at $\theta$ with radius $r$.

Theorem 2.2. Let $f \in L^{p}\left(\mathrm{R}^{n}\right) \cap L^{1}\left(\mathrm{R}^{n}\right)$ and let $P$ be an approximate identity such that (2.2) holds with $c_{3}=0, c_{4}=\infty$ and arbitrary constants $c_{1}, c_{2}>0$. Then there exists a constant $C$, independent of $f$, such that

$$
f^{*}(\theta)<C\left(f_{H L}(\theta)+\left(\left(|f|^{p}\right)_{H L}(\theta)\right)^{1 / p}\right)
$$

for all $\theta \in \mathbf{R}^{n}$.

Proof. Let $\chi(S, \cdot)$ denote the characteristic function of a set $S$. We can clearly assume that $\theta=0$. Let $(x, r) \in \mathscr{A}(0)$ be fixed, let $J \in \mathrm{N}$ be arbitrary and set $\eta=u(r) / J$. Moreover let $J^{\prime}$ be the smallest integer such that $\left|L\left(r, \eta J^{\prime}\right)\right| \leq c_{1} \rho(r)$ and note that we then have $J^{\prime} \leq J$ by (2.2). If $j<J^{\prime}$ and

$$
y \in L(r, j \eta) \backslash L(r,(j+1) \eta)
$$

then clearly $j \eta<P(y, r) \leq(j+1) \eta$ which implies that

$$
0 \leq P(x-t, r)-\eta \sum_{j=1}^{J^{\prime}-1} \chi(L(r, j \eta), x-t)<\eta
$$

whenever $x-t \notin L\left(r, J^{\prime} \eta\right)$. Set $S(s)=\{t: x-t \in L(r, s)\}$ and note that

$$
\eta \sum_{j=1}^{J^{\prime}-1} \lambda(S(j \eta)) \leq \int P(x-t, r) d \lambda(t)=1 .
$$


We then have

$$
\begin{aligned}
|\hat{f}(x, r)|= & \left|\int P(x-t, r) f(t) d \lambda(t)\right| \\
\leq & \left|\int\left(P(x-t, r)-\eta \sum_{j=1}^{J^{\prime}-1} \chi(L(r, j \eta), x-t)\right) f(t) d \lambda(t)\right| \\
& +\left|\int \eta \sum_{j=1}^{J^{\prime}-1} \chi(L(r, j \eta), x-t) f(t) d \lambda(t)\right| \\
(2.5) \leq & \eta\|f\|_{1}+\left|\int_{S\left(J^{\prime} \eta\right)} P(x-t, r) f(t) d \lambda(t)\right|+\left|\eta \sum_{j=1}^{J^{\prime}-1} \int_{S(j \eta)} f(t) d \lambda(t)\right|
\end{aligned}
$$

First we will estimate the second term. Set $B_{n}=\lambda(B(0,1))$.

$$
\begin{aligned}
& \mid \int_{S\left(J^{\prime} \eta\right)} P(x-t, r) f(t) d \lambda(t) \mid \\
& \quad \leq\|P(\cdot, r)\|_{q}\left(\int_{B\left(0,\left|S\left(J^{\prime} \eta\right)\right|\right.}|f(t)|^{p} d \lambda(t)\right)^{1 / p} \\
& \quad \leq\|P(\cdot, r)\|_{q}\left(\left(|f|^{p}\right)_{H L}(0) B_{n}\left(\left|S\left(J^{\prime} \eta\right)\right|\right)^{n}\right)^{1 / p} \\
& \leq\|P(\cdot, r)\|_{q}\left(\left(|f|^{p}\right)_{H L}(0)\right)^{1 / p} B_{n}^{1 / p}\left(\left(a+c_{1}\right) \rho(r)\right)^{n / p} \\
& \quad=c_{5}\left(\left(|f|^{p}\right)_{H L}(0)\right)^{1 / p}
\end{aligned}
$$

by (2.1) and the choice of $J^{\prime}$, where $c_{5}=B_{n}^{1 / p}\left(a+c_{1}\right)^{n / p}$

We now turn to the third term. Note that for $0 \leq s \leq\left(J^{\prime}-1\right) \eta$ we have $|L(r, s)|>c_{1} \rho(r)$ by the choice of $J^{\prime}$ and the assumption that $c_{3}=0$. For such $s$ we thus get

$$
|S(s)| \leq|L(r, s)|+|x| \leq\left(1+a c_{2}^{-1}\right)|L(r, s)|
$$

which together with (2.2) implies that

$$
|S(s)|^{n} \leq\left(1+a c_{2}^{-1}\right)^{n} c_{2} \lambda(L(r, s))=c_{6} \lambda(S(s)),
$$

where we have set $c_{6}=\left(1+a c_{2}^{-1}\right)^{n} c_{2}$. This combined with (2.4) gives

$$
\eta \sum_{j=1}^{J^{\prime}-1}|S(j \eta)|^{n} \leq c_{6}
$$


SO

$$
\begin{aligned}
\left|\eta \sum_{j=1}^{J^{\prime}-1} \int_{S(j \eta)} f(t) d \lambda(t)\right| & \leq \eta \sum_{j=1}^{J^{\prime}-1} \int_{B(0,|S(j \eta)|)}|f(t)| d \lambda(t) \\
& \leq \eta f_{H L}(0) \sum_{j=1}^{J^{\prime}-1} B_{n}|S(j \eta)|^{n} \\
& \leq B_{n} c_{6} f_{H L}(0)
\end{aligned}
$$

By (2.5) and the fact that $\eta$ can be taken arbitrarily small we obtain the inequality

$$
|\hat{f}(x, r)| \leq B_{n} c_{6} f_{H L}(0)+c_{5}\left(\left(|f|^{p}\right)_{H L}(0)\right)^{1 / p},
$$

and the proof is complete.

Proof of Theorem 2.1. By (1.3) it is easily seen that it is sufficient to prove the theorem for the function $f(\cdot) \chi(B(0, R), \cdot)$ where $R>0$ is arbitrary but fixed. Moreover a short compactness argument shows that $f(\cdot) \chi(B(0, R), \cdot) \in$ $L^{p}$, so we may assume that $f \in L^{p}$ and has compact support. It is also clear that we may assume that $c_{4}=\infty$.

Set $E(r)=\left\{x \in \mathrm{R}^{n}: P(x, r) \leq c_{3}\right\}$ and define functions $P_{1}$ and $P_{2}$ via

$$
P_{1}(r, x)=P(r, x) \chi(E(r), x) \quad \text { and } \quad P_{2}(r, x)=P(r, x) \chi\left(\mathrm{R}^{n} \backslash E(r), x\right) .
$$

Using the fact that $\lim _{r \rightarrow 0} \int_{B(\theta, r)}|f|=0$ for all $\theta \in \mathrm{R}^{n}$ and the properties (1.2) and (1.3) of $P$, it is easily seen that

$$
\lim _{\substack{r \rightarrow 0 \\ x \rightarrow \theta}} \int_{\mathrm{R}^{n}} P_{1}(x-t, r) f(t) d \lambda(t)=0
$$

and

$$
\lim _{r \rightarrow 0}\left\|P_{1}(\cdot, r)\right\|_{1}=0
$$

Hence the equality

$$
\lim _{\substack{(x, r) \in \mathscr{A}(\theta) \\ r \rightarrow 0}} \int_{\mathrm{R}^{n}} P(x-t, r) f(t) d \lambda(t)=f(\theta)
$$

holds if and only if

$$
\lim _{\substack{(x, r) \in \mathscr{A}(\theta) \\ r \rightarrow 0}} \int_{\mathrm{R}^{n}} \frac{P_{2}(x-t, r)}{\left\|P_{2}(\cdot, r)\right\|_{1}} f(t) d \lambda(t)=f(\theta)
$$

does. But $\frac{P_{2}(\cdot, r)}{\left\|P_{2}(\cdot, r)\right\|_{1}}$ is an approximate identity that satisfies condition (2.2) with $c_{3}=0$. Thus we may assume that $f$ and $P$ are such that Theorem 2.2 applies. 
From this point the proof follows standard arguments which we will only outline. Set

$$
h_{f}(\theta)=\lim _{R \rightarrow 0} \sup _{\substack{(x, r) \in \mathscr{A}(\theta) \\ r<R}}|\hat{f}(x, r)-f(\theta)|
$$

If $g$ is a continuous function with compact support we get

$$
\begin{aligned}
\left|h_{f}(\theta)\right| & \leq|f(\theta)-g(\theta)|+(f-g)^{*}(\theta) \\
& \leq|f(\theta)-g(\theta)|+C(|f-g|)_{H L}(\theta)+C\left(\left(|f-g|^{p}\right)_{H L}(\theta)\right)^{1 / p} .
\end{aligned}
$$

Fix $\epsilon>0$. Since the Hardy-Littlewood maximal function is weak $(1,1)$, we have that

$$
\lambda\left(\left\{\theta: h_{f}(\theta)>\epsilon\right\}\right)<C^{\prime}\left(\|f-g\|_{1}+\left\||f-g|^{p}\right\|_{1}\right)
$$

where $C^{\prime}$ is some constant which depends on $\epsilon$ but not on $g$. But $g$ can be chosen such that the right hand side is arbitrarily small and thus $\lambda\left(\left\{\theta: h_{f}(\theta)>\epsilon\right\}\right)=$ 0 . As $\epsilon$ was arbitrary, we are done.

REMARK. It is possible to give a direct proof of Theorem 2.1 largely based on the inequalities of the proof of Theorem 2.2. This gives a slightly stronger statement, because the proof then gives conditions on the points $\theta$ at which $\hat{f}(x, r)=(P(\cdot, r) * f)(x)$ has the desired limit. For $p=1$ the condition is simply that $\theta$ should be a Lebesgue point for $f$.

Another way to improve Theorem 2.1 is by the following simple observation.

Proposition 2.3. Let $f \in L^{1}\left(\mathrm{R}^{n}\right)$ and let $P_{1}, P_{2}$ be approximate identities such that $P_{2}$ is bounded above by $P_{1}$, and let $\rho: \mathbf{R}^{+} \rightarrow \mathbf{R}^{+}$be some function. Define

$$
\hat{f}_{i}(x, r)=\left(P_{i}(\cdot, r) * f\right)(x)=\int_{\mathrm{R}^{n}} P_{i}(x-t, r) f(t) d \lambda(t)
$$

and set

$$
\mathscr{A}(\theta)=\{(x, r):|x-\theta|<\rho(r)\} .
$$

If

$$
\lim _{\substack{r \rightarrow 0 \\(x, r) \in \mathscr{A}(\theta)}} \hat{f}_{1}(x, r)=f(\theta)
$$

for a.e. $\theta \in \mathbf{R}^{n}$, then the same is true for $\hat{f}_{2}$. 
Proof. Let $c>0$ be such that $P_{2}<c P_{1}$ and let $g \in L^{1}$ be continuous. Then

$$
\begin{aligned}
\lim _{R \rightarrow 0} & \sup _{\substack{x, r) \in \mathscr{A}(\theta) \\
r<R}}\left|\hat{f}_{2}(x, r)-f(\theta)\right| \\
& =\lim _{R \rightarrow 0} \sup _{\substack{(x, r) \in \mathscr{A}(\theta) \\
r<R}}\left|\left(P_{2}(\cdot, r) * f\right)(x)-f(\theta)\right| \\
& \leq|f(\theta)-g(\theta)|+\lim \sup \left|\left(P_{2}(\cdot, r) *(f-g)\right)(x)\right| \\
& <|f(\theta)-g(\theta)|+c \lim \sup \left|\left(P_{1}(\cdot, r) *|f-g|\right)(x)\right| \\
& \stackrel{\text { a.e. }}{=}(c+1)|f(\theta)-g(\theta)|,
\end{aligned}
$$

which implies that the integral of the first expression, (as a function of $\theta$ ), is 0 .

Theorem 2.1 can easily be reformulated for the torus $\mathbf{T}^{n}$. Let $m$ denote the normalized Haar measure on the group $\mathrm{T}^{n}$, let $f$ be a function in $L^{1}\left(\mathrm{~T}^{n}, m\right)$ and let $P: \mathrm{T}^{n} \times[0,1) \rightarrow \mathrm{R}^{+}$be an approximate identity on $\mathrm{T}^{n}$, which here means a positive function such that $\int_{\mathrm{T}^{n}} P(\cdot, r) d m=1$ for all $r$ and such that for any open set $\Omega \subset \mathrm{T}^{n}$ containing $1, P(\cdot, r)$ converge to 0 in $L^{\infty}\left(\mathrm{T}^{n} \backslash \Omega\right)$ as $r \rightarrow 1$. We wish to study the boundary behavior of

$$
\hat{f}\left(r e^{i t}\right)=\int_{[-\pi, \pi]^{n}} P\left(e^{i(t-s)}, r\right) f\left(e^{i s}\right) \frac{d \lambda(s)}{(2 \pi)^{n}},
$$

where we use the notation $e^{i s}=\left(e^{i s_{1}}, \ldots, e^{i s_{n}}\right)$.

To do this, we define $\tilde{f} \in L^{1}\left(\mathrm{R}^{n}\right)$ by $\tilde{f}(t)=f\left(e^{i t}\right)$ for $t \in[-\pi, \pi]^{n}$ and $\tilde{f}(t)=0$ otherwise. Similarly, define $\tilde{P}: \mathbf{R}^{n} \times(0,1] \rightarrow \mathbf{R}^{+}$by

$$
\tilde{P}(t, r)=(2 \pi)^{-n} P\left(e^{i t}, 1-r\right)
$$

for $t \in[-\pi, \pi]^{n}$ and $\tilde{P}(t, r)=0$ otherwise. Extend $\tilde{P}$ to $\mathrm{R}^{n} \times \mathrm{R}^{+}$by setting $\tilde{P}(\cdot, r)=\tilde{P}(\cdot, 1)$ for $r>1$. Then $\tilde{P}$ is clearly an approximate identity for $\mathrm{R}^{n}$ and we may apply Theorem 2.1 to $\tilde{f}$ and $\tilde{P}$. The following proposition, whose short proof we omit, transforms the conclusion to $\hat{f}$.

Proposition 2.4. Let $f, \tilde{f}, P, \tilde{P}$ be as above, let $\xi=e^{i \theta} \in \mathrm{T}^{n}$ be fixed with $\theta \in(-\pi, \pi)^{n}$ and let $\rho: \mathbf{R}^{+} \rightarrow \mathbf{R}^{+}$be given. Then

$$
\hat{f}\left(r e^{i t}\right)=\int_{[-\pi, \pi]^{n}} P\left(e^{i(t-s)}, r\right) f\left(e^{i s}\right) \frac{d \lambda(s)}{(2 \pi)^{n}}
$$

has the limit $f(\xi)$ when $|t-\theta| \leq \rho(1-r)$ and $r \rightarrow 1$ if and only if

$$
\int_{\mathrm{R}^{n}} \tilde{P}(t-s, r) \tilde{f}(s) d \lambda(s)
$$


has the limit $\tilde{f}(\theta)$ when $|t-\theta| \leq \rho(r)$ and $r \rightarrow 0$.

\section{Applications and further results}

In section I.6 of [5] there are considered approximate identities of the special type

$$
P_{h}(x, r)=\frac{1}{r^{n}} h\left(\frac{|x|}{r}\right),
$$

where $h$ is some decreasing positive function on $\mathbf{R}^{+}$such that

$$
\left\|P_{h}(\cdot, r)\right\|_{1}=1 .
$$

It is shown that for any $f \in L^{1}\left(\mathbf{R}^{n}\right)$ and a.e. $\theta \in \mathbf{R}^{n}$ the function

$$
\hat{f}(x, r)=\left(P_{h}(\cdot, r) * f\right)(x)=\int_{\mathrm{R}^{n}} P_{h}(x-t, r) f(t) d \lambda(t)
$$

has the non-tangential limit $f(\theta)$. In this section we will consider a more general class of approximate identities and show that the regions of convergence given by Theorem 2.1 cannot be improved.

Let $1 \leq p<\infty$ and let $q$ be its conjugate index. Let $h$ be a positive measurable function on $\mathrm{R}^{+}$and let $0<R \leq \infty$ be some fixed number. Set

$$
\iota(r)=\int_{B(0, R)} \frac{1}{r^{n}} h\left(\frac{|x|}{r}\right) d \lambda(x)=n B_{n} \int_{0}^{R / r} h(t) t^{n-1} d t,
$$

where $B_{n}$ is the volume of $B(0,1) \subset \mathrm{R}^{n}$. We shall assume that $h$ and $R$ are such that $0<\iota(r)<\infty$ for all $r>0$. Define the function $P_{h, R}: \mathrm{R}^{n} \times \mathrm{R}^{+} \rightarrow \mathrm{R}^{+}$ by

$$
P_{h, R}(x, r)=\chi(B(0, R), x) \frac{1}{\iota(r) r^{n}} h\left(\frac{|x|}{r}\right) .
$$

The aim is to show that if $P_{h, R}$ is an approximate identity (that satisfies (2.2) and $h$ satisfies (3.2) below) then the regions of convergence are completely determined by the function $\iota$.

In order to simplify the formulas we will assume that

$$
\int_{0}^{\infty} h^{q}(t) t^{n-1} d t<\infty
$$

if $q<\infty$ and $\sup _{t>0}|h(t)|<\infty$ if $q=\infty$. Then

$$
\begin{aligned}
\left\|P_{h, R}(\cdot, r)\right\|_{q} & =\frac{1}{\iota(r) r^{n}}\left(\int_{B(0, R)} h^{q}\left(\frac{|x|}{r}\right) d \lambda(x)\right)^{1 / q} \\
& =\frac{1}{\iota(r) r^{n}}\left(n B_{n} r^{n} \int_{0}^{R / r} h^{q}(t) t^{n-1} d t\right)^{1 / q} \sim \frac{1}{r^{n(1-1 / q)} \iota(r)}
\end{aligned}
$$


for small $r$ so that

$$
\rho(r) \sim r \iota^{p / n}(r)
$$

holds for small $r$, where $\rho$ as before is defined by (2.1).

If now $h$ and $R$ are such that $P_{h, R}$ is an approximate identity that satisfies (2.2) and such that (3.2) holds, then Theorem 2.1 applies and the estimate above shows that the regions of convergence given by Theorem 2.1 are effectively larger than the non-tangential ones if and only if

$$
\int_{0}^{\infty} h(t) t^{n-1} d t=\infty
$$

and $R<\infty$. We also see that these regions are unaffected by the size of $R$.

The next theorem summarizes our conclusions and moreover says that these regions are optimal.

THEOREM 3.1. Let $f \in L_{l o c}^{p}\left(\mathrm{R}^{n}\right) \cap L^{1}\left(\mathrm{R}^{n}\right)$, let $h$ be a positive function on $\mathrm{R}^{+}$such that (3.2) holds and let $0<R \leq \infty$ be a constant. Assume that $P_{h, R}$ is an approximate identity such that (2.2) holds. Then for a.e. $\theta \in \mathrm{R}^{n}$ the function

$$
\hat{f}(x, r)=\left(P_{h, R}(\cdot, r) * f\right)(x)=\int_{\mathrm{R}^{n}} P_{h, R}(x-t, r) f(t) d \lambda(t)
$$

has the limit $f(\theta)$ when $r \rightarrow 0$ and $x \rightarrow \theta$ inside the region

$$
\mathscr{A}(\theta)=\left\{(x, r):|x-\theta|<\operatorname{arl}^{p / n}(r)\right\}
$$

where $a$ is any fixed constant. Moreover, these regions are optimal in the sense that if $\gamma$ is any function such that the conclusion above holds with $\operatorname{arl}^{p / n}(r)$ replaced with $\gamma$, then $\gamma(r)=O\left(r \iota^{p / n}(r)\right)$ when $r \rightarrow 0$.

Proof. The first part is immediate by the remarks before the theorem. We turn to the second part. Let $\gamma$ be any function such that for a.e. $\theta$,

$$
\hat{f}(x, r)=\int_{\mathrm{R}^{n}} P_{h, R}(x-t, r) f(t) d \lambda(t)
$$

has the limit $f(\theta)$ when $r \rightarrow 0$ and $x \rightarrow \theta$ inside the region

$$
\mathscr{A}(\theta)=\{(x, r):|x-\theta|<\gamma(r)\} .
$$

If we do not have $\gamma(r)=O\left(r \iota^{p / n}(r)\right)$ when $r \rightarrow 0$, then we can take a sequence $\left(r_{k}\right)$ converging to 0 such that $r_{k}<R$ and

$$
\frac{r_{k} \iota^{p / n}\left(r_{k}\right)}{\gamma\left(r_{k}\right)}<2^{-k p / n}
$$


We will show that this yields a contradiction. Let $f_{k}$ be the function on $\mathbf{R}^{n}$ defined by $f_{k}(x)=\iota\left(r_{k}\right) \chi\left(B\left(0, r_{k}\right), x\right)$. Then

$$
\hat{f}_{k}\left(0, r_{k}\right)=\int_{\mathrm{R}^{n}} P_{h, R}\left(t, r_{k}\right) f_{k}(t) d \lambda(t)=n B_{n} \int_{0}^{1} h(s) s^{n-1} d s=d
$$

where $d$ is a constant that does not depend on $k$. Moreover

$$
\left\|f_{k}\right\|_{p}^{p}=B_{n} r_{k}^{n} \iota^{p}\left(r_{k}\right) .
$$

It is easy to show that there are constants $c$ and $C$ which only depend on $n$ such that for any $s \in(0,1)$ there are points $p_{1}, \ldots, p_{N}$ that satisfy

(i) $N<C / s^{n}$,

(ii) $\left|p_{i}-p_{j}\right|>c s$ whenever $i \neq j$,

(iii) $B(0,1) \subset \cup B\left(p_{i}, s\right)$.

Pick such points for $s=\gamma\left(r_{k}\right)$ and set

$$
g_{k}(\cdot)=\sum_{i} f_{k}\left(p_{i}-\cdot\right)=\iota\left(r_{k}\right) \sum_{i} \chi\left(B\left(p_{i}, r_{k}\right), \cdot\right) .
$$

By (iii) we have that

$$
\sup _{|x-\theta|<\gamma\left(r_{k}\right)} \hat{g}_{k}\left(x, r_{k}\right) \geq d
$$

for all $\theta \in B(0,1)$. Moreover, $r_{k} / \gamma\left(r_{k}\right) \rightarrow 0$ (by (3.4)) so it follows by (ii) that when $k$ is big enough the supports of the terms in (3.5) are mutually disjoint. For such $k$ we have the estimate

$$
\left\|g_{k}\right\|_{p}<\left(\frac{C}{\left(\gamma\left(r_{k}\right)\right)^{n}} B_{n} r_{k}^{n} \iota^{p}\left(r_{k}\right)\right)^{1 / p}<\left(C B_{n}\right)^{1 / p} 2^{-k} .
$$

Set $G_{K}=\sum_{k \geq K} g_{k}$. Then $G_{K} \in L^{p}\left(\mathrm{R}^{n}\right)$ and

$$
\lim _{r \rightarrow 0} \sup _{\substack{(x, s) \in \mathscr{A}(\theta) \\ s<r}} \hat{G}_{K}(x, s)>d
$$

for all $\theta \in B(0,1)$, independently of $K$, so $G_{K}(\theta)>d$ for a.e. $\theta \in B(0,1)$. But at the same time we have $\lim _{K \rightarrow \infty}\left\|G_{K}\right\|_{p}=0$ by (3.6), a contradiction.

Given a function $P_{h, R}$ it might be difficult to check wether Theorems 2.1 and 3.1 apply. We now give a simple criterion on $h$ which is sufficient for $P_{h, R}$ to be an approximate identity such that (2.2) holds. 
Proposition 3.2. Let $h$ be a positive function on $\mathrm{R}^{+}$such that (3.2) holds and let $0<R \leq \infty$ be a constant. Assume that $\exists K \geq 0$ such that $h(t) t^{n-1}$ is decreasing for $t \geq K$. Then $P_{h, R}$ is an approximate identity such that (2.2) holds if and only if

$$
\lim _{r \rightarrow 0} \frac{1}{\iota(r) r^{n}} h\left(\frac{1}{r}\right)=0
$$

Proof. It is clear that $P_{h, R}$ satisfies (1.2). Since $h(t)$ is decreasing for $t \geq K$, the first part of (1.3) holds if and only if $h$ satisfies

$$
\lim _{r \rightarrow 0} \frac{1}{\iota(r) r^{n}} h\left(\frac{c}{r}\right)=0, \quad \forall c>0 .
$$

Moreover, the fact that $h(t) t^{n-1}$ is decreasing for $t \geq K$ implies that $\iota(c r) \sim$ $\iota(r)$ for small $r$ and thus (3.7) and (3.8) are equivalent. The second part of (1.3) holds if and only if

$$
\lim _{r \rightarrow 0} \int_{B(0, c)} P_{h, R}(\cdot, r) d \lambda=1
$$

for $c<R$. But

$$
\int_{B(0, c)} P_{h, R}(\cdot, r) d \lambda=\frac{n B_{n}}{\iota(r)} \cdot \int_{0}^{c / r} h(t) t^{n-1} d t=1-\frac{n B_{n}}{\iota(r)} \cdot \int_{c / r}^{R / r} h(t) t^{n-1} d t,
$$

and thus (3.9) holds if and only if

$$
\lim _{r \rightarrow 0} \int_{c / r}^{R / r} \frac{h(t) t^{n-1}}{\iota(r)} d t=0,
$$

which is indeed the case by (3.8) and the estimate

$$
\int_{c / r}^{R / r} \frac{h(t) t^{n-1}}{\iota(r)} d t \leq \frac{R-c}{r} \frac{h(c / r)(c / r)^{n-1}}{\iota(r)} .
$$

which holds for $r<c / K$. We have now shown that that $P_{h, R}$ is an approximate identity if and only if (3.7) holds. It remains to show that (2.2) holds.

First assume that $h$ does not have compact support. Pick $K^{\prime}>K$, set $c_{4}=R / 2 K^{\prime}$ and

$$
u(r)=\frac{h\left(K^{\prime}\right)}{\iota(r) r^{n}} .
$$

Note that for $r<c_{4}$ we have

$$
L(r, u(r))=\left\{x \in \mathrm{R}^{n}: h(|x| / r)>h\left(K^{\prime}\right)\right\}=r\left\{x \in \mathrm{R}^{n}: h(|x|)>h\left(K^{\prime}\right)\right\}
$$


so $|L(r, u(r))| \leq K^{\prime} r$. The fact that $\rho(r) \sim r \iota^{p / n}(r)$ for small $r$ and that $\iota$ is a decreasing function implies that there exist $c_{1}>0$ such that $|L(r, u(r))|<$ $c_{1} \rho(r)$ for $r<c_{4}$.

Now fix $s, r$ such that $r<c_{4}$ and $s<u(r)$. Put $t=|L(r, s)| / r$ and note that $t \geq K^{\prime}$ and

$$
L(r, s) \supset B(0, r t) \backslash B(0, r K) .
$$

Therefore

$$
\frac{|L(r, s)|^{n}}{\lambda(L(r, s))} \leq \frac{t^{n}}{B_{n}\left(t^{n}-K^{n}\right)} \leq \frac{K^{\prime n}}{B_{n}\left(K^{\prime n}-K^{n}\right)}
$$

so (2.2) holds with $c_{1}, c_{4}$ as above and $c_{2}=\frac{K^{\prime n}}{B_{n}\left(K^{n}-K^{n}\right)}, c_{3}=0$.

The case when $h$ has compact support follows with a similar argument. We omit the details.

We will now show that Theorem 1.2 by Sjögren and Rönning follows directly from Theorem 3.1. Let $P_{1 / 2}$ be the normalized square root of the Poissonkernel, i.e. $P_{1 / 2}: \mathrm{T} \times[0,1) \rightarrow \mathrm{R}^{+}$is defined via

$$
P_{1 / 2}(\xi, r)=\frac{(P(\xi, r))^{1 / 2}}{\left\|(P(\cdot, r))^{1 / 2}\right\|_{1}}
$$

where $P(\xi, r)=\frac{1-r^{2}}{|\xi-r|^{2}}$ denotes the Poisson-kernel.

Corollary 3.3. Let $f \in L^{p}(\mathrm{~T}), 1 \leq p<\infty$, and set

$$
\hat{f}\left(r e^{i t}\right)=\int_{0}^{2 \pi} P_{1 / 2}\left(e^{i(t-s)}, r\right) f\left(e^{i s}\right) \frac{d s}{2 \pi} .
$$

Also let a be arbitrary and set $\gamma(r)=a(1-r)\left(\log \frac{1}{1-r}\right)^{p}$. Then, for a.e. $\theta \in \mathrm{T}$ we have that $\hat{f}(z) \rightarrow f(\theta)$ when $z \rightarrow \theta$ inside

$$
z \in \mathscr{A}(\theta)=\left\{r e^{i s}:|\arg \theta-s|<\gamma(r)\right\} .
$$

Moreover these regions of convergence cannot be improved.

Proof. By standard arguments it is easily shown that

$$
\frac{1-r^{2}}{\left|e^{i t}-r\right|^{2}} \sim \frac{1-r}{t^{2}+(1-r)^{2}}
$$

for $t \in[-\pi, \pi]$ and $0<r<1$. Set $h(t)=\left(1+t^{2}\right)^{-1 / 2}$ and recall the construction of $\tilde{P}_{1 / 2}$ given before Proposition 2.4. It follows that $\tilde{P}_{1 / 2} \sim P_{h, \pi}$ and that $\iota(r) \sim \log \frac{1}{r}$ for small $r$. The corollary is now an immediate application of Propositions 2.3, 2.4 and Theorem 3.1. 
The above result is due to Sjögren [4] in the case $p=1$ and due to Rönning [2] for $p>1$. A recently published article by Brundin [1] gives simpler proofs of these results. All proofs are rather complicated and rely on estimates using the actual shape of the kernel $P_{1 / 2}$. As the above corollary shows, the result can be proved without any knowledge of $P_{1 / 2}$ except the estimate $\left\|P_{1 / 2}(\cdot, r)\right\|_{q}^{p} \sim$ $\left((1-r) \log \left(\frac{1}{1-r}\right)^{p}\right)^{-1}$ for $r \rightarrow 1^{-}$and some basic observations.

Let $0<\alpha<1$. We may of course also consider the normalized $\alpha$ th root of the Poisson-kernel, denoted $P_{\alpha}$. If we carry out the above scheme in this situation, the corresponding function $h$ will be $h(t)=\left(1+t^{2}\right)^{-\alpha}$. For $\alpha>$ $1 / 2, h \in L^{1}$ and hence $\iota(r) \sim 1$ for small $r$, which explains why we get the "usual" non-tangential regions in this case. For $\alpha<1 / 2$ the function $h$ does not satisfy condition (3.7), that is, $P_{\alpha}$ are not approximate identities for $\alpha<1 / 2$.

We will now construct an example related to condition (2.2). It can be shown that the conclusion of Theorem 1.3 cannot hold for all approximate identities, but an interesting question is wether (2.2) can be removed for the approximate identities considered in this section. In other words, if $h$ is such that $P_{h, R}$ is an approximate identity that does not satisfy condition (2.2), is the conclusion of Theorem 1.3 still true?

We are not able to answer this question. However, the stronger form of Theorem 2.1 that was indicated in the remark after its proof implies that for each $f \in L^{1}$ the equality

$$
\lim _{\substack{r \rightarrow 0 \\(x, r) \in \mathscr{A}(\theta)}} \hat{f}(x, r)=f(\theta)
$$

actually holds at all Lebesgue-points $\theta$ of $f$. This result does not hold for all approximate identities of the form $P_{h, R}$.

To verify this claim, we shall construct a function $h \in L^{1}\left(\mathrm{R}^{+}\right)$and a function $f \in L^{1}(\mathrm{R})$ such that:

(i) 0 is a Lebesgue point for $f$ and $f(0)=0$.

(ii) $h$ is such that $P_{h, \infty}$ is an approximate identity.

(iii) $\hat{f}(0, r)$ does not go to zero as $r \rightarrow 0$.

Moreover, we will show that $P_{h, \infty}$ fails condition (2.2) by very little, in the sense that given any function $\sigma$ that satisfies $\lim _{r \rightarrow 0} \sigma(r)=\infty, h$ can be taken such that the function

$$
\tau(r)=\sup _{s \geq 1} \frac{|L(r, s)|}{\lambda(L(r, s))}
$$

satisfies $\tau(r)=O(\sigma(r))$ as $r \rightarrow 0$. 
We begin with the construction of $f$ and $h$ that satisfies (i)-(iii). Let $A_{k}$ denote the intervals $\left[2^{-k}-4^{-k}, 2^{-k}\right]$ and set

$$
f=\sum_{k=1}^{\infty} \frac{2^{k}}{k} \chi\left(A_{k}, \cdot\right) .
$$

It is easily seen that

$$
\limsup _{x \rightarrow 0^{+}} \frac{\int_{-x}^{x}|f(t)| d t}{2 x} \leq \limsup _{m \rightarrow \infty} 2^{m-1} \int_{0}^{2^{-m}} f(t) d t \leq \limsup _{m \rightarrow \infty} \frac{1}{m}=0
$$

so 0 is a Lebesgue point for $f$.

We turn to the construction of $h$. Note that for a positive $h \in L^{1}\left(\mathrm{R}^{+}\right)$, a necessary and sufficient condition for $P_{h, \infty}$ to be an approximate identity is

$$
\lim _{R \rightarrow \infty} \sup _{x \geq R} R h(x)=0 .
$$

Let $k(m)$ be numbers such that

$$
\sum_{k=1}^{k(m)} \frac{1}{k} \geq m^{2}
$$

let $d_{m}$ satisfy

$$
d_{m}\left(2^{-k(m)}-4^{-k(m)}\right)>d_{m-1},
$$

and set

$$
h(x)=\sum_{m=1}^{\infty} \frac{1}{d_{m} m^{2}} \sum_{k=1}^{k(m)} 2^{k} \chi\left(A_{k}, \frac{x}{d_{m}}\right)
$$

for $x \geq 0$. Note that $m$ th term of the first sum has support in the interval

$$
\left[d_{m}\left(2^{-k(m)}-4^{-k(m)}\right), d_{m}\right],
$$

so by (3.11) the supports of the terms are disjoint. Moreover the $L^{1}$-norm of the $m$ th term is less than $1 / m^{2}$ so $h \in L^{1}\left(\mathbf{R}^{+}\right)$.

We thus get $\iota(r)=\left(2\|h\|_{1}\right)$. The function $\sum_{k=1}^{\infty} 2^{k} \chi\left(A_{k}, x\right)$ stays below the curve $1 / x$ which implies that the $m$ th term stays below $-1 /\left(m^{2} x\right)$. This in turn implies that (3.10) is fulfilled so $P_{h, \infty}=\left(2\|h\|_{1}\right)^{-1} \frac{1}{r} h\left(\frac{|x|}{r}\right)$ is an approximate identity. 
It remains to verify (iii), which follows from the calculation

$$
2\|h\|_{1} \hat{f}\left(0, \frac{1}{d_{m}}\right) \geq \int_{\mathrm{R}} f(t) \frac{1}{m^{2}} \sum_{k=1}^{k(m)} 2^{k} \chi\left(A_{k},|-t|\right) d t=\frac{1}{m^{2}} \sum_{k=1}^{k(m)} \frac{1}{k} \geq 1 .
$$

Thus not even the radial limit of $\hat{f}$ exists at 0 , and hence the approximate identity $P_{h, \infty}$ cannot satisfy condition (2.2). We now show that $h$ can be chosen such that

$$
\tau(r)=\sup _{s \geq 1} \frac{|L(r, s)|}{\lambda(L(r, s))}
$$

satisfies $\tau(r)=O(\sigma(r))$ when $r \rightarrow 0$, where $\sigma$ is any given function that satisfies $\lim _{r \rightarrow 0} \sigma(r)=\infty$.

First note that $\tau$ is a decreasing function of $r$ that depends on the choice of $h$ and thus on the constants $d_{m}$. These can be chosen arbitrarily as long as equation (3.11) is satisfied. Moreover $\|h\|_{1}$ is independent of the sequence $\left(d_{m}\right)$, and a quite complicated calculation (which we omit) shows that

$$
\tau(r) \leq 2^{k(m-1)}, \quad \forall r>\left(m^{2} 2\|h\|_{1} d_{m} 2^{-k(m)}\right)^{-1} .
$$

From this it follows that we can choose $\left(d_{m}\right)$ such that $\tau(r)<\sigma(r)$ for sufficiently small $r$.

\section{Products of approximate identities}

Let $P_{1}(x, r)=\frac{1}{\pi} \frac{r}{\left(x^{2}+r^{2}\right)}$ be the Poisson kernel for $\mathrm{R}$ and consider the function on $\mathrm{R}^{n}$ defined by

$$
P(x, r)=P_{1}\left(x_{1}, r_{1}\right) P_{1}\left(x_{2}, r_{2}\right) \cdots P_{1}\left(x_{n}, r_{n}\right),
$$

where as usual it is implicitly understood that $x=\left(x_{1}, \ldots, x_{n}\right)$ etc. The boundary behavior of convolutions of a function $f$ with $P$ was studied by Zygmund (see [6], Ch. XVII). It follows from his results that if $f \in L^{p}\left(\mathrm{R}^{n}\right)$ has compact support and $p>1$, then for a.e. $\theta$ the extension

$$
\hat{f}(x, r)=\int_{\mathrm{R}^{n}} P(x-t, r) f(t) d \lambda(t)
$$

has the limit $f(\theta)$ when $(x, r) \rightarrow(\theta, 0)$ inside the set

$$
\mathscr{A}(\theta)=\left\{(x, r):\left|x_{i}-\theta_{i}\right|<a r_{i}\right\},
$$

where $a$ is any constant. If, however, $f \in L^{1}\left(\mathrm{R}^{n}\right)$ then the above result is in general false. This is well known and intimately connected with the fact 
that $L^{1}\left(\mathrm{R}^{n}\right)$-functions are not in general strongly differentiable. In fact, the above statement can easily be proved using the result in [3] concerning strong differentiability.

Note that the situation is quite different from the previous sections. First of all the function $P$ is not an approximate identity. It has too many variables and moreover $P(\cdot, r)$ is not small outside a ball around 0 for small $|r|$. Another difficulty is that the level sets of $P$ do not satisfy a condition like (2.2), even though the level sets of $P_{1}$ are intervals.

We will now consider arbitrary functions $P$ that arise in the above way as products of approximate identities on R. At the end of this section we shall give a generalization to $\mathrm{D}^{n}$ of Theorem 1.2 by Sjögren and Rönning, which also can be considered as a complement to Theorem 2 in Brundin [1].

Let $P_{i}, i=1, \ldots, n$, be approximate identities on $\mathrm{R}$, let $\rho_{i}$ be the corresponding functions given by $\rho_{i}(r)=\left\|P_{i}(\cdot, r)\right\|_{q}^{-p / n}$, and assume that (2.2) holds for each $P_{i}$. Let the function $P$ be given by

$$
P(x, r)=P_{1}\left(x_{1}, r_{1}\right) P_{2}\left(x_{2}, r_{2}\right) \cdots P_{n}\left(x_{n}, r_{n}\right) .
$$

The main result is the following.

Theorem 4.1. Let $1 \leq p<\infty$ be given and let $P$ be defined as above. Let $\epsilon>0$, let $f \in L^{p+\epsilon}\left(\mathrm{R}^{n}\right)$ be a function with compact support and set

$$
\mathscr{A}(\theta)=\left\{(x, r):\left|x_{i}-\theta_{i}\right|<a \rho_{i}\left(r_{i}\right)\right\}
$$

where $a$ is any fixed constant. Then for a.e. $\theta \in \mathbf{R}^{n}$ the function

$$
\hat{f}(x, r)=(P(\cdot, r) * f)(x)=\int_{\mathrm{R}^{n}} P(x-t, r) f(t) d \lambda(t)
$$

has the limit $f(\theta)$ when $(x, r) \rightarrow(\theta, 0)$ inside the region $\mathscr{A}(\theta)$.

REMARK. As noted above, the theorem is false for functions $f \in L^{1}\left(\mathrm{R}^{n}\right)$. Thus we may not in general allow $\epsilon=0$.

Proof. Assume first that (2.2) holds for each $P_{i}$ under the additional assumption that $c_{3}=0$ and $c_{4}=\infty$. If $f$ is a measurable function on $\mathbf{R}^{n}$ we set

$$
f_{H L}^{i}(x)=\sup _{r>0} \frac{1}{2 r} \int_{x_{i}-r}^{x_{i}+r} f\left(x_{1}, \ldots, x_{i-1}, t, x_{i+1}, \ldots, x_{n}\right) d t
$$

and define the (sublinear) operators $M_{1}, \ldots, M_{n}$ on $L^{p+\epsilon}\left(\mathrm{R}^{n}\right)$ via

$$
M_{i}(f)(x)=\left(f_{H L}^{i}(x)+\left(\left(|f|^{p}\right)_{H L}^{i}(x)\right)^{1 / p}\right) .
$$


Since the Hardy-Littlewood maximal operator is bounded on both spaces $L^{p}(\mathrm{R})$ and $L^{1+\epsilon / p}(\mathrm{R})$, it follows that $M_{i}$ is a bounded operator on $L^{p+\epsilon}\left(\mathrm{R}^{n}\right)$. By Theorem 2.2 there exists a constant $C$ such that whenever $\left|x_{i}-\theta_{i}\right|<a \rho_{i}\left(r_{i}\right)$ we have

$$
\left|\int_{\mathrm{R}} P_{i}\left(x_{i}-t, r_{i}\right) f\left(\theta_{1}, \ldots, \theta_{i-1}, t, \theta_{i+1}, \ldots, \theta_{n}\right) d t\right| \leq C M_{i}(f)(\theta) .
$$

By repeated use of the above formula and Fubini's Theorem we conclude that for $(x, r) \in \mathscr{A}(\theta)$ we have

$$
|\hat{f}(x, r)|=\left|\int_{\mathrm{R}^{n}} P(x-t, r) f(t) d \lambda(t)\right| \leq C^{n} M_{1}\left(M_{2}\left(\ldots M_{n}(f)\right)\right)(\theta) .
$$

Let $C_{1}$ be such that $\left\|M_{i}\right\| \leq C_{1}$ and as in section 2 let $f^{*}$ denote the maximal function of $f$ with respect to the regions $\mathscr{A}(\cdot)$. Then

$$
\left\|f^{*}\right\|_{p+\epsilon} \leq C^{n} C_{1}^{n}\|f\|_{p+\epsilon} .
$$

The desired conclusion now follows as in the proof of Theorem 2.1. Finally, the assumptions that $c_{3}=0$ and $c_{4}=\infty$ may be removed by similar methods as in the proof of Theorem 2.1, we omit the details.

As an application of the above theorem we will generalize Corollary 3.3. Consider the function $P: \mathrm{T}^{n} \times[0,1)^{n} \rightarrow \mathrm{R}^{+}$given by

$$
P(\xi, r)=P_{1 / 2}\left(\xi_{1}, r_{1}\right) P_{1 / 2}\left(\xi_{2}, r_{2}\right) \ldots P_{1 / 2}\left(\xi_{n}, r_{n}\right)
$$

and the corresponding extension to $\mathrm{D}^{n}$ of a function $f \in L^{p}\left(\mathrm{~T}^{n}\right)$ given by

$$
\hat{f}\left(\left(r_{1} e^{i t_{1}}, \ldots, r_{n} e^{i t_{n}}\right)\right)=\int_{[-\pi, \pi]^{n}} P\left(e^{i(t-s)}, r\right) f\left(e^{i s}\right) \frac{d \lambda(s)}{(2 \pi)^{n}} .
$$

Corollary 4.2. Let $1 \leq p<\infty, \epsilon>0$ be given and $f \in L^{p+\epsilon}\left(\mathrm{T}^{n}\right)$. Let a be arbitrary and set $\rho(r)=a(1-r)\left(\log \frac{1}{1-r}\right)^{p}$. Then, for a.e. $\xi \in \mathrm{T}^{n}$, $\hat{f}(z) \rightarrow f(\xi)$ when $z \rightarrow \xi$ and

$$
z \in \mathscr{A}(\xi)=\left\{\left(r_{1} e^{i t_{1}}, \ldots, r_{n} e^{i t_{n}}\right) \in \mathrm{D}^{n}:\left|\arg \xi_{k}-t_{k}\right|<\rho\left(r_{k}\right)\right\} .
$$

Proof. This follows immediately from Theorem 4.1 by using the same techniques as in the proof of Corollary 3.3 and the appropriate versions of Propositions 2.3 and 2.4.

Remark. By considering $P_{\alpha}$ for $\alpha>1 / 2$ we get the above corollary but with $\rho(r)=a(1-r)$. 
Corollary 4.2 seems to be new and should be compared with Theorem 2 of [1], where the same result is proved under the assumption that $f \in L^{\infty}\left(T^{n}\right)$ and with $\rho$ such that $\rho(r)=O\left((1-r)^{\alpha}\right)$ for all $\alpha<1$. Note that this holds for the functions $a(1-r)\left(\log \frac{1}{1-r}\right)^{p}$. See also [2] for a related result.

\section{REFERENCES}

1. Brundin, M., Approach regions for $L^{p}$ potentials with respect to the square root of the Posson kernel, to appear in Math. Scand.

2. Rönning, J., Convergence results for the square root of the Poisson kernel, Math. Scand. 81(2) (1997), 219-235.

3. Saks, S., On the strong derivatives of functions of intervals, Fund. Math. 25 (1935), 245-252.

4. Sjögren, P., Une remarque sur la convergence des fonctions propres du laplacien à valeur propre critique, Theoris du potentiel (Orsay, 1983), 544-548. Springer, Berlin, 1984.

5. Stein, E. M., Harmonic Analysis, Princeton University Press, 1993.

6. Zygmund, A., Trigonometric Series, Cambridge University Press, 1959.

CENTRE FOR MATHEMATICAL SCIENCES

LUND UNIVERSITY

BOX 118

SE-221 00 LUND

SWEDEN

E-mail: mc@maths.lth.se 